\section{Patrolling monocytes promote kidney disease}

The treatment of lupus nephritis with B cell-targeted therapies has so far been mostly unsuccessful, despite a wealth of evidence implicating autoantibodies and immune complexes in the pathogenesis of systemic lupus erythematosus (SLE). The results of a new study suggest that rather than being autoantibody-mediated, early lupus nephritis might instead be promoted by 'patrolling monocytes.'

"In previous work, we identified the protein TNIP1 as part of the Toll-like receptor signalling complex and established gene-deficient mice to study its function in vivo," explains corresponding author Hans Häcker. "We found that these mice spontaneously developed a lupus-like disease characterized by glomerulonephritis, autoreactive antibodies and overall reduced survival." TNIP1 was subsequently confirmed as an SLE-susceptibility locus, making Tnip1 $1^{-/}$mice a good model for human SLE.

Häcker and colleagues found that Tnip1 $1^{-/} \mathrm{Rag}^{-/-}$mice, which lack mature T cells and B cells, still developed glomerulonephritis, indicating that adaptive immune system responses were not necessary for kidney pathology. Turning instead to the innate immune system, the researchers discovered that LY6C ${ }^{\text {lo }}$ patrolling monocytes (also known as non-classical monocytes) accumulated in the kidneys of Tnip $1^{-/-}$mice, and also in the kidneys of mice with two other models of lupus (MRL/lpr mice and B6.Sleyaa mice). CD $14^{\mathrm{dim}} \mathrm{CD} 16^{+}$monocytes, the equivalent population in humans, were also present in increased numbers in the glomeruli of patients with lupus nephritis.

Genetic deletion of patrolling monocytes in Tnip $1^{-/-}$mice abrogated glomerulonephritis, but did not

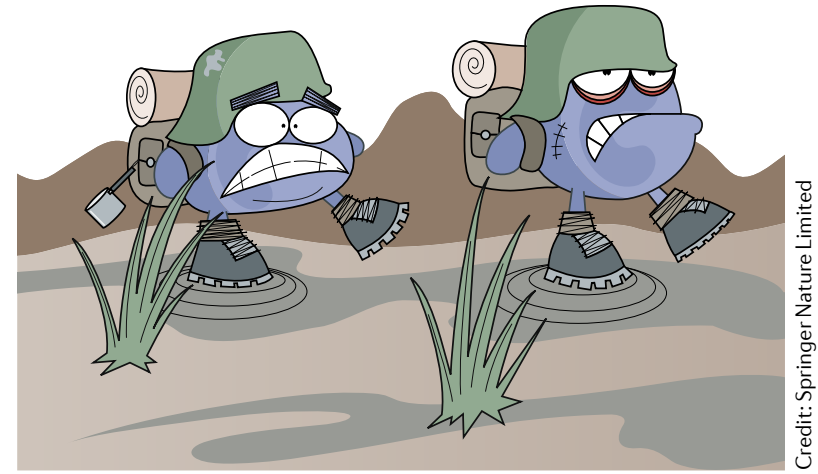

affect autoantibody production or other disease parameters.

"Collectively, these data suggest that different disease symptoms in SLE are mediated by different immune mechanisms," says Häcker. "The early phase of glomerulonephritis seems to be promoted by patrolling monocytes rather than autoreactive antibodies and immune complexes, patrolling monocytes ... accumulated in the kidneys of Tnip 1/-- mice whereas other, more systemic disease parameters proceed independently of patrolling monocytes," he concludes.

Joanna Collison

ORIGINAL ARTICLE Kuriakose, J et al. Patrolling monocytes promote the pathogenesis of early lupus-like glomerulonephritis. J. Clin. Invest. https://doi.org/10.1172/JCl125116 (2019)

\section{Engineered fusion protein disrupts CD40 signalling}

The CD40 pathway has long been an attractive therapeutic target for treating autoimmune diseases; however, early clinical trials of monoclonal antibodies targeting CD40 ligand (CD40L) were halted owing to platelet-related thromboembolic complications. Findings from a new study suggest that VIB4920, a bivalent CD40L-specific Tn3 fusion protein, could suppress

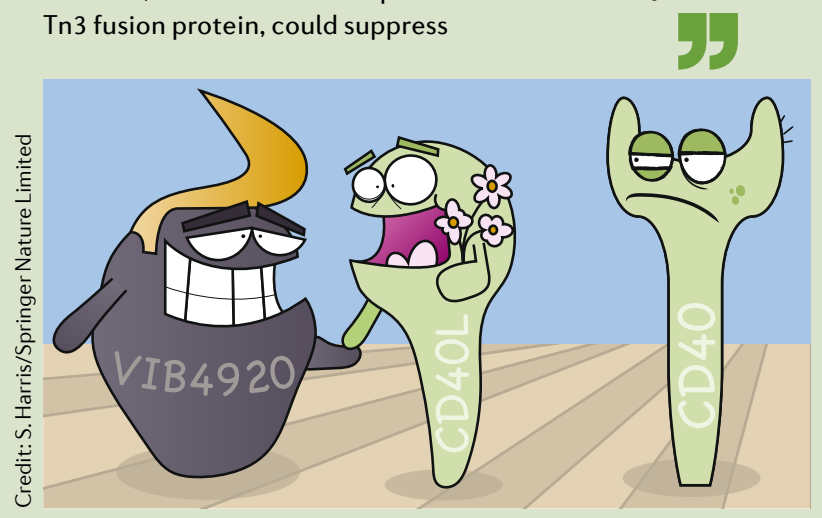

treatment with high-dose ... VIB4920 led to improvements in disease activity

the CD40-CD40L axis while avoiding these safety concerns.

VIB4920 blocks the interaction between CD40 and CD40L by competing with CD40. "This novel construct maintains the specificity of an antibody-based therapeutic, demonstrating specific high-affinity binding to $\mathrm{CD} 40 \mathrm{~L}$, but lacks an $\mathrm{Fc}$ domain," explains Jodi Karnell, first author of the paper. "As the Fc domain of anti-CD40L antibodies was definitively linked to the safety issues observed in early clinical trials, VIB4920 is predicted to be a safer approach for targeting this pathway."

In preclinical studies, VIB4920 blocked CD40L signalling as well as differentiation and activation of human $B$ cells, but did not induce platelet aggregation in vitro. In healthy volunteers, VIB4920 was well-tolerated and inhibited the humoral immune response to immunization with keyhole limpet hemocyanin in a dose-dependent manner.

In a 12-week phase lb trial in patients with active rheumatoid arthritis (RA), treatment with high-dose $(1,000 \mathrm{mg}$ or $1,500 \mathrm{mg}$ every other week) VIB4920 led to improvements in disease activity as well as reductions in titres of rheumatoid factor autoantibodies and biomarkers related to immune activation. "The results in patients with RA provide a clear demonstration of the potential of a non-antibody based CD40L-targeted therapeutic to provide benefit to patients with autoimmune disease," says corresponding author Jörn Drappa. "Most importantly, we did not see any evidence of platelet activation or any thromboembolic events to date."

Encouraged by these early results, the researchers are planning clinical trials of VIB4920 in various autoimmune and inflammatory diseases in which the CD40-CD40L pathway is implicated.

\section{Sarah Onuora}

ORIGINAL ARTICLE Karnell, J. L. et al. A CD40Ltargeting protein reduces autoantibodies

and improves disease activity in patients with autoimmunity. Sci. Transl. Med. 11, eaar6584 (2019) 